\title{
INEQUALITIES OF JOHN-NIRENBERG TYPE IN DOUBLING SPACES
}

\author{
Stephen M. BuCKLEY
}

Abstract. The concept of an $\mathrm{H}$-chain set in a doubling space $X$, which generalizes that of a Hölder domain in Euclidean space, is defined and investigated. We show that every H-chain set is mean porous, and that its outer layer has measure bounded by a power of its thickness. As a consequence, we show that a John-Nirenberg type inequality holds on an open subset $\Omega$ of $X$ if, and often only if, $\Omega$ is an H-chain set.

\section{Introduction}

Suppose that $\Omega \subset \mathbb{R}^{n}$ is open. A function $u: \Omega \rightarrow \mathbb{R}$ lies in $B M O(\Omega)$ if either of the following equivalent conditions is satisfied:

$$
\begin{aligned}
\|u\|_{B M O(\Omega)} & =\sup _{Q \subset \Omega}|Q|^{-1} \int_{Q}\left|u-u_{Q}\right| d x<\infty \\
\|u\|_{B M O_{\mathrm{loc}}(\Omega)} & =\sup _{2 Q \subset \Omega}|Q|^{-1} \int_{Q}\left|u-u_{Q}\right| d x<\infty
\end{aligned}
$$

where $Q$ is an arbitrary cube and $2 Q$ is its concentric double dilate. We write $u \in E I(\Omega)$ if it satisfies the integrability condition

$$
\exists C \in \mathbb{R}: \quad|\Omega|^{-1} \int_{\Omega} \exp \left(\left|u-u_{\Omega}\right| / C\right) d x \leq 16,
$$

and we define $\|u\|_{E I(\Omega)}$ to be the smallest $C$ for which this condition holds; the peculiar " 16 " on the right is merely for later convenience (a comparable norm is obtained by replacing it by any fixed factor larger than 1).

A theorem of Reimann and Rychener $[R R]$ says that $(0.1)$ and $(0.2)$ are equivalent for any $\Omega$ and that the associated "norms" are comparable. The classical theorem of John and Nirenberg [JN] tells us that when $\Omega$ is a cube, $E I(\Omega) \subset B M O(\Omega)$. More generally, Smith and Stegenga [SS2] and Hurri-Syrjänen [H] showed that $B M O(\Omega)$ embeds in $E I(\Omega)$ if and only if $\Omega$ is a Hölder domain (as defined in Section $3^{1}$ ). Related Euclidean results can be found in [G1] and [S]. In this

1991 Mathematics Subject Classification. 43A85, 26D15.

The author was supported in part by Enterprise Ireland. Part of this paper was written while the author was visiting the University of Jyväskylä in 1998; he wishes to thank the Mathematics Department there for its hospitality. 
paper, we generalize these results to many doubling spaces $(X, d, \mu)$. To do this, we replace cubes by metric balls, and Lebesgue measure by the doubling measure $\mu$ in the definitions of $B M O(\Omega)$, $B M O_{\text {loc }}(\Omega)$, and $E I(\Omega)$. Since Euclidean cubes are balls with respect to the $l^{\infty}$ metric, these new spaces generalize the classical ones (the switch from cubes to balls also leaves unchanged spaces such as classical $\left.B M O\left(\mathbb{R}^{n}\right)\right)$.

It is easy to construct metric spaces on which the two function spaces defined by generalizing (0.1) and (0.2) are unequal (see Example 5.1), but we shall see that they are equal for length spaces (in the sense of Gromov, as defined in Section 3). We are more interested though in John-Nirenberg type results, i.e. results which say that all (restrictions of) functions in $B M O(\Omega)$ lie in $E I\left(\Omega^{\prime}\right)$, where $\Omega^{\prime} \subset \Omega$ are fixed open subsets of $X$. Specifically, we look at two cases: the global case where $\Omega=X$, and $\Omega^{\prime}=B$, a metric ball, and the local case where $\Omega=\Omega^{\prime}$ is a bounded proper subset of $X$.

Investigating the local case is our main goal, but we first adapt classical arguments to prove a global version that we shall need to use in the local case. By Jones' extension theorem [J], uniform domains (including balls) in the Euclidean-Lebesgue setting are BMO extension domains, so the local version for balls follows immediately from the global case. Vodop'yanov and Greshnov [VG] extended this to show that uniform domains in a complete length space are BMO extension domains; the authors show that balls in Heisenberg groups are uniform domains, but this rather special result requires considerable effort.

The problem with this approach is that uniformity, although the right condition for BMO extendability, is a much stronger condition than what is needed to prove John-Nirenberg. A uniform ball is transversely path-connected in a nice way, whereas to prove exponential integrability, it will suffice to assume much less, namely that the set $\Omega$ is an $H$-chain set, which roughly means that there exists a "fairly short" chain of balls from any $x \in \Omega$ to a fixed $x_{0} \in \Omega$. H-chain sets generalize the notion of Hölder domains but are not necessarily connected (see Section 3 ); in a length space, all balls are H-chain sets.

Let us briefly comment on a fallacious argument that may seem to suggest that the local result for balls follows easily from the global one, namely that since a ball $B_{0}$ in a doubling space is "obviously" a doubling subspace of $X$ (i.e. it is a doubling space when equipped with the restricted metric and measure), the global result for the space $B_{0}$ gives the local result for the ball $B_{0}$. There are two distinct fallacies here. First, the "obvious fact" that a ball is a doubling space (as used in [RL], for instance) is not in general true, although it can be shown to be true if $X$ has a length metric. Counterexamples are not hard to construct; see Section 5. Secondly, even if this "fact" is valid, there are usually $B$-balls that are not $X$-subballs of the set $B$ (the prefix indicates the ambient space). Consequently, $B M O(B)$ may be a stronger condition if we treat $B$ as a doubling space in its own right than if $B$ is merely a ball in a larger space $X$. We give an explicit example in Section 5 where local John-Nirenberg fails even though all balls are doubling subspaces.

Our main imbedding results are most easily stated in the context of length spaces; this we now do. These theorems generalise the above-mentioned results of [RR], [JN], [SS2], and [H]; the necessary definitions are given in Sections 1 and 3.

\footnotetext{
${ }^{1}$ For now, suffice it to say that a simply-connected planar domain $\Omega$ is a Hölder domain if and only if the Riemann mapping from the unit disk to $\Omega$ is uniformly Hölder continuous.
} 
Theorem 0.3. Suppose $(X, d, \mu)$ is a doubling space and $d$ is a length metric. Then $B M O_{\mathrm{loc}}(\Omega)=$ $B M O(\Omega)$ for every open $\Omega \subset X$. Furthermore, $\|\cdot\|_{B M O(\Omega)} \leq C\|\cdot\|_{B M O_{\mathrm{loc}}(\Omega)}$ for some constant $C$ dependent only on the doubling constant of $\mu$.

Theorem 0.4. Suppose $(X, d, \mu)$ is a doubling space and $d$ is a length metric. Then $B M O(\Omega) \subset$ $E I(\Omega)$ for every Hölder domain $\Omega \subset X$. Furthermore,

$$
\|\cdot\|_{E I(\Omega)} \leq C\|\cdot\|_{B M O(\Omega)}
$$

for some constant $C$ dependent only on the doubling constant of $\mu$ and the Hölder constant of $\Omega$. In particular, all metric balls support this imbedding with a constant $C$ dependent only on the doubling constant. Conversely the imbedding (0.5) fails if $\Omega$ is not a Hölder domain.

We further refine Theorem 0.4 and related results by introducing a subspace $G B M O(\Omega)$ ("Good BMO") of $B M O(\Omega)$, which equals $B M O(\Omega)$ if the local John-Nirenberg result is true. Thus John-Nirenberg splits into two parts, $B M O(\Omega)=G B M O(\Omega)$ and $G B M O(\Omega) \subset E I(\Omega)$; neither part has to be true in a general doubling space (see Section 5), even if $\Omega$ is a ball. In the classical setting, the first part is quite trivial but, interestingly, only this "trivial" part requires the H-chain condition to work in a general space. The "non-trivial" part follows under a much weaker assumption $O L D(\Omega)$ which roughly says that the measure of the boundary layer of $\Omega$ decays like some positive power of its thickness.

Even in the Euclidean setting with a doubling measure, local John-Nirenberg for balls cannot be proved by a straightforward adaptation of classical arguments. The otherwise excellent book of Heinonen, Kilpeläinen, and Martio [HKM, Appendix II], contains a faulty proof of such a JohnNirenberg inequality; we are grateful to Juha Heinonen for pointing out this error. The problem is that, unlike a cube, a ball cannot be nicely subdivided into balls of similar size, and so the classical-type proof must use balls that are only partially contained in $B_{0}$. Theorem 0.4 fixes this error; the above-mentioned result of Vodop'yanov and Greshnov also solves the global-to-local problem for balls in a Euclidean or Heisenberg group setting.

After the preliminary material of Section 1, we prove the global results in Section 2. Section 3 examines H-chain sets, mean porosity, and outer layer decay; in particular we generalize a result of Koskela and Rohde [KR] by proving that the boundary of an H-chain set is mean porous. Section 4 proves that H-chain and OLD conditions are sufficient for the two parts of local JohnNirenberg. Finally, Section 5 contains some counterexamples, and shows that the H-chain and OLD conditions are often necessary.

I wish to thank Tasuhiro Gotoh for pointing out the short proofs of Proposition 3.2 and Lemma 3.5 given here.

\section{Definitions and notation}

Suppose that $(X, d)$ is a metric space. If $z \in X, r>0$, the ball $B(z, r)$ (or $B_{X}(z, r)$ if the space needs to be specified) is the set of all $y \in X$ such that $d(z, y)<r$; for us a "ball" is always such an open metric ball. If $B$ is a ball, we denote its radius and center by $r(B)$ and $z(B)$ respectively, and write $t B, t>0$, for its concentric dilate $B(z(B), \operatorname{tr}(B))$. Note that in a metric space, the equality $B\left(z_{1}, r_{1}\right)=B\left(z_{2}, r_{2}\right)$ does not allow us to deduce that $z_{1}=z_{2}$ or that $r_{1}=r_{2}$. Consequently, when we say that $B$ is a ball, it is implicitly assumed that we 
are also specifying $z(B)$ and $r(B)$. With this convention, the notation $t B$ is well-defined in any metric space. Whenever $\Omega$ is a proper open subset of a metric space, we define $d_{\Omega}: \Omega \rightarrow \mathbb{R}$ by the equation $d_{\Omega}(x)=\operatorname{dist}(x, X \backslash \Omega)$; we shall also write, for instance, $d_{\Omega}^{-1}((r, R])$ in place of $\{x \in \Omega: r<\operatorname{dist}(x, X \backslash \Omega) \leq R\}$.

A positive Borel measure $\mu$ on a metric space $(X, d)$ is said to be doubling if there exists a constant $C$ such that $0<\mu(2 B) \leq C \mu(B)<\infty$ for every ball $B$ in $X$. We say that $(X, d, \mu)$ is a doubling space if $(X, d)$ is a metric space and $\mu$ is a doubling measure on $X$. A doubling space is a special case of a homogeneous space in the sense of Coifman and Weiss [CW1]; these latter spaces merely have a quasimetric rather than a metric. We refer the reader to [CW1] for an exposition of analysis on homogeneous spaces, and to $[\mathrm{HK}]$ for much more on doubling spaces.

The doubling condition is equivalent to the slightly more convenient condition that $\mu\left(B_{1}\right) \leq$ $C \mu\left(B_{2}\right)$ whenever $B_{2} \subset B_{1}$ are balls and $r\left(B_{2}\right) \geq r\left(B_{1}\right) / 2$. We define the doubling constant of $\mu, C_{\mu}$, to be the smallest value of $C \geq 1$ for which this last condition is valid. We shall often refer to $X$ as a doubling space, with the understanding that there is an attached metric $d$ and doubling measure $\mu$. If $S \subset X$ has finite positive measure and $u: S \rightarrow \mathbb{R}$, we write $u_{S}=f_{S} u d \mu=\mu(S)^{-1} \int_{S} u d \mu$.

The following covering lemma follows from [CW1, III.1.2]; note that we can choose $k=5$ in that result since $d$ is a genuine metric.

Lemma 1.1. Suppose $U \subset X$, where $U$ is open and $X$ is a doubling space. If $U$ is covered by a family $\mathcal{B}$ of balls of uniformly bounded radius, then there exists a countable pairwise disjoint family $\left\{B_{i}\right\}_{i \in S} \subset \mathcal{B}$ such that $\bigcup_{i \in S} 5 B_{i} \supset U$.

Let $\Omega$ be an open subset of a doubling space $X$. We now define the subspaces of $L_{\text {loc }}^{1}(\Omega)$ that we call $B M O(\Omega), B M O_{\text {loc }}(\Omega)$, and $E I(\Omega)$. First, $u \in B M O(\Omega)$ (resp. $\left.u \in B M O_{\text {loc }}(\Omega)\right)$ if there exists a constant $C$ such that $f_{B}\left|u-u_{B}\right| d \mu \leq C$ for every ball $B \subset \Omega$ (resp. $2 B \subset \Omega$ ); $\|u\|_{B M O(\Omega)}$ (resp. $\left.\|u\|_{B M O_{\mathrm{loc}}(\Omega)}\right)$ is the smallest $C$ for which this condition holds. Next, $u \in E I(\Omega)$ if there exists a positive constant $C$ such that

$$
\exists C \in \mathbb{R}: \quad f_{\Omega} \exp \left(\left|u-u_{\Omega}\right| / C\right) d \mu \leq 16 ;
$$

$\|u\|_{E I(\Omega)}$ is the smallest $C$ for which this condition holds. In general these spaces depend on both the metric and the measure but it is interesting to note that in the Euclidean setting with $\Omega$ being a cube, $B M O(\Omega)$ gives the same space for any measure associated with a weight in the Muckenhoupt class $A_{\infty}(Q)$; see [RR], [G1].

\section{Global John-Nirenberg}

In a footnote on page 594 of [CW2], it is mentioned that a global John-Nirenberg inequality holds in any doubling space, but this is not proved (it is proved in [HS] for doubling graphs). In this section, we give such a proof. In fact we prove a little more, namely that if $u \in B M O\left(2 B_{0}\right)$, then $u \in E I\left(B_{0}\right)$, for any ball $B_{0}$ in a doubling space; this will prove useful in the local case. Throughout this section, $(X, d, \mu)$ is a doubling space, $C_{\mu}$ is its doubling constant, and $B_{0}=$ $B\left(z_{0}, r_{0}\right)$ is an arbitrary but fixed ball in $X$. 
Suppose that $B_{i} \subset \Omega \subset X, 0 \leq i \leq k$, are balls such that $r\left(B_{i}\right) / r\left(B_{i-1}\right) \in[1 / 2,2]$, and either $B_{i-1}$ contains $B_{i}$, or $B_{i}$ contains $B_{i-1}$, for all $1 \leq i \leq k$. We call such a set of balls a nesting chain of length $k$ in $\Omega$ from $B_{0}$ to $B_{k}$. If $u \in B M O\left(2 B_{0}\right)$, and $\left\{B_{i}: 0 \leq i \leq k\right\}$ is any nesting chain in $2 B_{0}$, it readily follows from the doubling condition that $\left|u_{B_{i}}-u_{B_{i-1}}\right| \leq C_{\mu}\|u\|_{B M O\left(2 B_{0}\right)}$. For instance, if $B_{i-1} \subset B_{i}$, then

$$
\left|u_{B_{i}}-u_{B_{i-1}}\right| \leq f_{B_{i-1}}\left|u-u_{B_{i}}\right| d \mu \leq C_{\mu} f_{B_{i}}\left|u-u_{B_{i}}\right| d \mu \leq C_{\mu}\|u\|_{B M O\left(2 B_{0}\right)} .
$$

Adding these inequalities for all $1 \leq i \leq k$ gives the following simple lemma.

Lemma 2.1. If $\left\{B_{i}: 0 \leq i \leq k\right\}$ is a nesting chain of length $k$ in $2 B_{0}$ from $B$ to $B^{\prime}$, and $u \in B M O\left(2 B_{0}\right)$, then $\left|u_{B}-u_{B^{\prime}}\right| \leq k C_{\mu}\|u\|_{B M O\left(2 B_{0}\right)}$.

We now state the main result of this section.

Theorem 2.2. If $u \in B M O\left(2 B_{0}\right)$, then $\|u\|_{E I\left(B_{0}\right)} \leq 6 C_{\mu}\|u\|_{B M O\left(2 B_{0}\right)}$.

The proof of this theorem, like that of the classical John-Nirenberg Theorem, proceeds by performing nested Calderón-Zygmund type decompositions at an arithmetic sequence of levels. We isolate this part of the proof in the following lemma, where metric balls $\left\{B_{j}^{m}\right\}$ take the place of the usual Calderón-Zygmund cubes at level $m$. In our variation of this argument, the covering and nesting properties are both valid only for dilates of the balls in the decompositions.

Lemma 2.3. Suppose that $u \in B M O\left(2 B_{0}\right), u_{B_{0}}=0$, and let $\alpha \geq 6 C_{\mu}\|u\|_{B M O\left(2 B_{0}\right)}$. Then there exist balls $B_{j}^{m}=B\left(z_{j}^{m}, r_{j}^{m}\right), j \in I^{m}, m \in \mathbb{N}$, where each $I^{m}$ is a countable index set, and for every $m \in \mathbb{N}, j \in I^{m}$ :

(i) $z_{j}^{m} \in B_{0}$ and $r_{j}^{m} \leq r_{0} / 16$;

(ii) $m \alpha<\left|u_{B_{j}^{m}}\right| \leq(m+1 / 6) \alpha$;

(iii) $(m-1 / 2) \alpha<\left|u_{8 B_{j}^{m}}\right| \leq m \alpha$;

(iv) $|u(x)| \leq m \alpha$, for almost all $x \in B_{0} \backslash \bigcup_{i \in I^{m}} 5 B_{i}^{m}$;

(v) if $m>1$, there exists $j^{\prime} \in I^{m-1}$ such that $8 B_{j}^{m} \subset 8 B_{j^{\prime}}^{m-1}$;

(vi) the balls $B_{i}^{m}, i \in I^{m}$, are pairwise disjoint.

Proof. By an induction on $i$, we shall define, for all $i, m \in \mathbb{N}$, families $\mathcal{G}_{i}^{m} \supset \mathcal{G}_{i-1}^{m}$ of balls with centers in $B_{0}$ and radius at most $r_{0} / 8$, from which we define a residual set $S_{i}^{m}=B_{0} \backslash \bigcup_{B \in \mathcal{G}_{i}^{m}} 5 B$. We begin the induction by setting $\mathcal{G}_{0}^{m}=\emptyset$. We next define some auxiliary families of balls for all $i>0$. Let

$$
\begin{aligned}
\mathcal{B}_{i}^{m} & =\left\{B\left(x, 2^{-i-3} r_{0}\right): x \in S_{i-1}^{m}\right\}, \\
\widetilde{\mathcal{B}}_{i}^{m} & =\left\{B \in \mathcal{B}_{i}^{m}: m \alpha<\left|u_{B_{j}^{m}}\right|\right\},
\end{aligned}
$$

and let $\mathcal{F}_{i}^{m}$ be a countable pairwise disjoint subset of $\widetilde{\mathcal{B}}_{i}^{m}$ such that the 5 -dilates of the balls in $\mathcal{F}_{i}^{m}$ cover the union of the balls in $\widetilde{\mathcal{B}}_{i}^{m}$; such a subset exists by Lemma 1.1. We complete the inductive step by defining $\mathcal{G}_{i}^{m}$ to be $\mathcal{G}_{i-1}^{m} \cup \mathcal{F}_{i}^{m}$. To finish our construction, we define $\mathcal{G}^{m}=\bigcup_{i=1}^{\infty} \mathcal{G}_{i}^{m}$, 
$S^{m}=\bigcap_{i=1}^{\infty} S_{i}^{m}$, and list all elements of $\mathcal{G}^{m}$ in the form $\left\{B_{j}^{m}\right\}$, where $j$ ranges over a countable index set $I^{m}$.

We need to show that the constructed collection of balls have properties (i)-(vi). First note that if $x \in B_{0}$ and $r \geq r_{0} / 8$, then there is a nested chain of length 3 in $2 B_{0}$ from $B(x, r)$ to $B\left(x, r_{0}\right)$. Appending $2 B_{0}$ and $B_{0}$ to this chain, we get a nested chain of length five from $B(x, r)$ to $B_{0}$. Thus

$$
\left|u_{B(x, r)}\right| \leq 5 C_{\mu}\|u\|_{B M O\left(2 B_{0}\right)}<\alpha, \quad x \in B_{0}, r \geq r_{0} / 8 .
$$

Statements (i) and the lower bound in (ii) follow immediately from the construction. The upper bound in (iii) holds by construction if $B_{j}^{m} \in \mathcal{F}_{i}^{m}$ for some $i>3$, and otherwise it is implied by (2.4); more generally, we see that $\left|u_{B}\right| \leq m \alpha$, whenever $B=2^{i} B_{j}^{m}, i \in \mathbb{N}$, and $2^{i} r_{j}^{m} \leq r_{0}$. Taking $B=2 B_{j}^{m}$, and using the estimate $\left|u_{B_{j}^{m}}-u_{2 B_{j}^{m}}\right| \leq C_{\mu}\|u\|_{B M O\left(2 B_{0}\right)}$, we deduce the upper bound in (ii). Using the lower bound in (ii) and the obvious nested chain of length three from $B_{j}^{m}$ to $8 B_{j}^{m}$, we get the lower bound in (iii).

To show (iv), it suffices to note that if $x \in B_{0} \cap S^{m}$ then $\left|u_{B\left(x, 2^{-i-2} r_{0}\right)}\right| \leq m \alpha$ for all $i \in \mathbf{N}$. Letting $i \rightarrow \infty$, and using the Lebesgue Differentiation Theorem for doubling spaces, we deduce (iv).

As for (v), let us suppose that $B_{j}^{m} \in \mathcal{F}_{i}^{m}$. The lower bound in (ii) implies that either $B_{j}^{m}$ is in $\widetilde{\mathcal{B}}_{i}^{m-1}$, or $z_{j}^{m} \in B_{0} \backslash S_{i-1}^{m-1}$, but the first possibility contradicts the upper bound in (ii). In fact, it is clear that we need a nested chain of length at least two to bridge this gap, and so $z_{j}^{m} \in B \in \mathcal{G}_{i-2}^{m-1}$. Since all of these balls have radius at least four times that of $B_{j}^{m}$, (v) follows readily.

Finally we must prove (vi). Suppose that that this is false. Let $B_{j}=B\left(z_{j}, r_{j}\right), j=1,2$, denote an intersecting pairs of balls in $\mathcal{G}^{m}$. Clearly $B_{1}$ and $B_{2}$ are not members of the same family $\mathcal{F}_{i}^{m}$ so we may assume that $B_{1} \in \mathcal{G}_{i}^{m}, B_{2} \in \mathcal{G}^{m} \backslash \mathcal{G}_{i}^{m}$ for some fixed $i>0$. Since $r\left(B_{2}\right)<r\left(B_{1}\right)$, $B_{2} \subset 5 B_{1}$, which contradicts the fact that $z_{2} \in S_{i}^{m}$, and so we are done.

Proof of Theorem 2.2. Normalize $u$ so that $u_{B_{0}}=0$ and let $\alpha=6 C_{\mu}\|u\|_{B M O\left(2 B_{0}\right)}$. It suffices to show that

$$
\mu\left(S_{m}\right) \equiv \mu\left(\left\{x \in B_{0}:|u(x)|>m \alpha\right\}\right) \leq \mu\left(B_{0}\right) / 2\left(3 C_{\mu}\right)^{m}, \quad m \in \mathbb{N} .
$$

To see this, we write $L_{m}=\left\{x \in B_{0}: m \alpha<|u(x)| \leq(m+1) \alpha\right\}$ if $m \in \mathbb{N}$, and $L_{0}=\left\{x \in B_{0}\right.$ : $|u(x)| \leq \alpha\}$. Using (2.5), we get

$$
\begin{aligned}
f_{B_{0}} \exp (|u| / \alpha) d \mu & \leq \frac{1}{\mu\left(B_{0}\right)}\left(\sum_{m=0}^{\infty} \int_{L_{m}} \exp (|u| / \alpha) d \mu\right) \\
& \leq e+\sum_{m=1}^{\infty} e^{m+1} \frac{\mu\left(S_{m}\right)}{\mu\left(B_{0}\right)} \leq e+\frac{e}{2} \cdot \sum_{m=1}^{\infty}\left(\frac{e}{3 C_{\mu}}\right)^{m} \\
& \leq e+\frac{e^{2}}{6 C_{\mu}\left(1-e / 3 C_{\mu}\right)} \leq e+\frac{e^{2}}{6(1-e / 3)}<16 .
\end{aligned}
$$

In the notation of Lemma 2.3, $S_{m} \subset U_{m} \equiv \bigcup_{j \in I^{1}} 8 B_{j}^{m}$. Since $u \in B M O\left(2 B_{0}\right)$, it readily follows that

$$
\mu\left(U_{1}\right) \leq \frac{\mu\left(2 B_{0}\right)\|u\|_{B M O\left(2 B_{0}\right)}}{\alpha} \leq \frac{\mu\left(B_{0}\right)}{6 C_{\mu}}
$$


which gives (2.5) for $m=1$. Next, let $B \equiv 8 B_{j}^{m}$ for some $j \in I^{m}, m \in \mathbb{N}$, and let

$$
\mathcal{F}(B)=\left\{8 B_{i}^{m+1}: i \in I^{m+1}, 8 B_{i}^{m+1} \subset B\right\}
$$

By Lemma 2.3(iii), $\left|u_{B}-u_{B^{\prime}}\right| \geq \alpha / 2$ for all $B^{\prime} \in \mathcal{F}(B)$. It follows from the $B M O\left(2 B_{0}\right)$ condition that $\mu\left(\bigcup_{B^{\prime} \in \mathcal{F}(B)} B^{\prime}\right) \leq \mu(B) / 3 C_{\mu}$. Summing over all $B$ and using the nesting property of these 8-dilates, we have $\mu\left(U_{m+1}\right) \leq \mu\left(U_{m}\right) / 3 C_{\mu}$. An easy induction argument now gives $(2.5)$ for all $m \in \mathbb{N}$.

\section{H-chains, Mean Porosity, and Outer Layer Decay}

In this section we define and examine the previously mentioned OLD and H-chain conditions. H-chain sets generalize the concept of Hölder domains (originally defined in [GM] as domains satisfying a "quasihyperbolic boundary condition") to the metric space setting, using chains of balls in place of rectifiable paths. The relationship between Hölder domains and H-chain sets is analogous to that between John domains and Boman chain sets (John domains and connected Boman chain sets in a doubling space are defined and discussed in [BKL2]). In each case, the path condition implies the corresponding chain condition, and this implication can be reversed in a length space (defined below). However, the chain conditions are satisfied by some disconnected and non-quasiconvex sets (sets lacking paths of length comparable to the distance between their endpoints). We shall prove that $\mathrm{H}$-chain sets are OLD and the closely related fact that the boundary of an H-chain set is mean porous, generalizing a result of Koskela and Rohde [KR].

A length space is a metric space $(X, d)$ in which the distance between any pair of points equals the infimum of the lengths of rectifiable paths joining them; we say that $d$ is a length metric. Many doubling spaces have length metrics; an important class of examples are spaces of Carnot-Carathéodory type (where distance is given as the infimum of lengths of "subunit" paths), including those spaces associated with Hörmander or Grushin families of vector fields. The recent literature on these and related spaces is quite extensive; see for instance [GN], [BKL1], [VSC], [NSW], and many of the references cited therein.

Suppose that $\Omega$ is a proper open subset of a doubling space $X$, of finite positive diameter $R$, and let $\Omega_{r}=d_{\Omega}^{-1}((0, r))$. Given $0<\delta \leq 1 \leq K<\infty$, we say that $\Omega$ is a $(\delta, K)-O L D$ set if $\mu\left(\Omega_{r}\right) \leq(K r / R)^{\delta} \mu(\Omega)$ for all $0<r<R$. We say that an open subset $G \subset X$ is a $(\delta, K)-B O L D$ set if every ball $B \subset G, B \neq X$, is a $(\delta, K)$-OLD set. Above, "OLD" stands for "outer layer decay"; the term "outer layer" is more accurate than "boundary layer" since, for small $r>0$, a point in $\Omega_{r}$ is close to $X \backslash \Omega$, but it may not be close to $\partial \Omega$-in fact, $\partial \Omega$ might even be empty.

For the remainder of this section, it is implicitly assumed that any ball contained in $\Omega$ has radius at most $2 \operatorname{diam}(\Omega)$. It is easy to construct spaces with gaps where this is false but this is not a real restriction since, when viewed as a set, any such oversized ball is all of $\Omega$ and equals the concentric ball of radius $2 \operatorname{diam}(\Omega)$.

Suppose that $J, K, L \in \mathbb{R}, J>1, K, L \geq 1$, and that $B_{*} \equiv B\left(z_{*}, r_{*}\right) \subset \Omega$, where $\Omega$ is a proper open subset in the metric space $(X, d)$ and $r_{*} \leq \operatorname{diam}(\Omega)$. We say that a chain of balls $B_{i} \equiv B\left(z_{i}, r_{i}\right) \subset \Omega, 0 \leq i \leq k$, is an H-chain (with respect to $\Omega$ and with parameters $J, L, B_{*}$ ) of length $k$ for $B(x, r) \subset \Omega, r \leq \operatorname{diam}(\Omega)$, if:

(a) $\left(z_{0}, r_{0}\right)=(x, r)$ and $\left(z_{k}, r_{k}\right)=\left(x_{*}, r_{*}\right)$; 
(b) if $0 \leq i<k$, then $1 / J \leq r_{i} / r_{i+1} \leq J$, and $B_{i} \cap B_{i+1}$ contains a ball $B_{i}^{\prime} \equiv B\left(z_{i}^{\prime}, r_{i}^{\prime}\right)$, $r_{i}^{\prime}=\left(r_{i}+r_{i+1}\right) / 2 J$

(c) $B\left(z_{i}, L r_{i}\right) \subset \Omega, 0<i<k$.

We say that $\Omega$ is an $H$-chain set, with parameters $J, K, L, B_{*}$, if every ball $B(x, r) \subset \Omega, r \leq$ $\operatorname{diam}(\Omega)$, possesses an H-chain of length at most $K \log _{2}\left(2 r_{*} / r\right)$. We say that an open subset $G$ of $X$ is a $B H$-chain set with parameters $J, K, L$ if all balls $B \subset G, B \neq X$, are H-chain sets with parameters $J, K, L$, and $B_{*} \equiv(1 / L) B$.

The condition for an H-chain set is invariant under multiplication of the metric by a positive constant. Obviously, the definition implies that $r_{*}$ is strictly within a factor 2 of the radius of the largest ball that fits inside $\Omega$, but the choice of $B_{*}$ is not otherwise crucial—if $B_{*}$ is changed to any other such large ball, the H-chain set still satisfies the new condition, perhaps with a larger parameter $K$. Clearly, the chain condition is stronger when $L$ is larger. In fact, one can give examples that are chain sets with $L=L_{1}$ but not with $L=L_{2}$, for any $1 \leq L_{1}<L_{2}$-it suffices to consider $\Omega=B(0,1) \subset X$, where $X$ is a subset of the Euclidean line having the form $\{0\} \cup \bigcup_{k=1}^{\infty}\left\{1+c^{-k}, 1-c^{-k}\right\}$ for a suitable value of $c \in(0,1)$. When the H-chain condition is used as a hypothesis in this paper, the choice of parameter $L=1$ usually suffices, but occasionally we need $L>1$. Consequently, when we show that certain classes of sets are H-chain sets, we shall remark that it is possible to take $L>1$ if this is the case, as it usually is.

A useful property of H-chains is that they can be spliced. If $\left\{B_{i}\right\}_{i=0}^{k}$ is an H-chain for a ball $B$, then for any $0<j<k$ we get another $\mathrm{H}$-chain (possibly longer or shorter) if we replace the balls $\left\{B_{i}\right\}_{i=j}^{k}$ by the balls in some H-chain of $B_{j}$. It follows that if $\left\{B_{i}\right\}_{i=0}^{k}$ is a minimal length $\mathrm{H}$-chain for a particular ball $B$, then $\left\{B_{i}\right\}_{i=j}^{k}$ is also a minimal length $\mathrm{H}$-chain for $B_{j}$, and that

$$
r_{i} \leq 2 r_{*} 2^{-(k-i) / K}, \quad 0 \leq i \leq k .
$$

BH-chain sets include all length spaces. For instance, the specific chains in the paragraph after Theorem 4.5 suffice to prove this with $J=2, K=L=1$ (one could instead take $L=2$, for instance, but then one needs to sacrifice the nesting property of that particular chain).

H-chain sets are closely connected with Hölder domains; these domains appear to have been defined only in Euclidean space, but can be generalized to a metric space setting: a proper open subset $\Omega$ in a metric space $(X, d)$, is a Hölder domain if there exists some constant $C$ such that for every $x \in \Omega$ we can find a path $\gamma_{x}$ joining $x$ to a fixed point $x_{*} \in \Omega$ such that

$$
\int_{\gamma_{x}} \frac{d s}{d_{\Omega}(x)} \leq C \log \left(\frac{C}{d_{\Omega}(x)}\right),
$$

where $d s$ is arclength measure. The reader may recognise the above integral as the quasihyperbolic length of $\gamma_{x}$; for more on Hölder domains and the quasihyperbolic metric in Euclidean spaces, see $[\mathrm{K}]$. The notion of a Hölder domain has proved useful in complex dynamics and elsewhere; see for example $[\mathrm{GS}]$.

A Hölder domain is always an H-chain set with $L=2$, say, since we can cover the Hölder path $\gamma_{x}$ by balls $B\left(\gamma_{x}\left(t_{i}\right), d_{\Omega}\left(\gamma_{x}\left(t_{i}\right)\right) / 2\right)$, where the quasihyperbolic distance between $\gamma_{x}\left(t_{i-1}\right)$ and $\gamma_{x}\left(t_{i}\right)$ is $1 / 100$, say, for each $i$ (although we allow the distance for $i=k$ to be smaller than this). Conversely, H-chain sets are Hölder domains in a length space, or in any other space where 
any pair of points in a ball can be joined in the ball by a path of length comparable with the ball's radius. In this case, we dilate the chain balls by a factor slightly less than 1 so that they are of uniformly bounded quasihyperbolic diameter but still overlap, and then we connect each point in $B_{i-1} \cap B_{i}$ with a point in $B_{i} \cap B_{i+1}$ by a path of length comparable with $r\left(B_{i}\right)$; the details are left to the reader.

Without some restriction, H-chain sets might not be Hölder domains. For example, if $X=$ $\mathbb{R}^{2} \backslash(\{1 / 2\} \times \mathbb{R})$ with the Euclidean metric attached, then $B_{X}((0,0), 1)$ is a disconnected H-chain ball. Alternatively, if $X$ is the real line with the metric $d(x, y)=|x-y|^{p}$ for some $0<p<1$, then $X$ is a BH-chain set with no non-constant rectifiable paths. The interested reader is invited to construct (as can be done) a BH-chain space $X$ which has a non-Hölder ball, although every pair of points in this ball can be connected by a rectifiable path (without length control, of course).

The bound on the length of an H-chain implies that an H-chain set $\Omega$ contains no large ball, i.e. no ball of radius $r>2 r_{*}$. On the other hand, chain balls for $B(x, r)$ must have radius at least $c\left(r / r_{*}\right)^{N} r_{*}$, for some positive $c, N$. We may for instance take $N=K \log _{2} J$ and $c=J^{-K}$, but we cannot in general take $N=1$. In the Euclidean plane, for example, let us define $\Omega$ to be the union of a central square $S_{0}$ connected to a sequence of smaller squares $S_{j}, j \in \mathbb{N}$, via narrow square necks $T_{j}$, defined as follows:

$$
\begin{aligned}
& S_{0}=\left\{(x, y) \in \mathbb{R}^{2}:|x|<2,|y|<2\right\}, \\
& S_{j}=\left\{(x, y) \in \mathbb{R}^{2}:\left|x-a_{j}\right|<s_{j},\left|y-2-2 t_{j}-s_{j}\right|<s_{j}\right\}, \quad j \in \mathbb{N}, \\
& T_{j}=\left\{(x, y) \in \mathbb{R}^{2}:\left|x-a_{j}\right|<t_{j},\left|y-2-t_{j}\right| \leq t_{j}\right\}, \quad j \in \mathbb{N},
\end{aligned}
$$

where $a_{j}=2^{-j+1}, s_{j}=2^{-j-1}$, and $t_{j}=2^{-N j}$. Taking $B_{*}=B(0,1)$, it is not hard to show that $\Omega$ is an H-chain set (with $J=L=2$, and $K=K(N)$ ). However if $z=\left(a_{j}, 2+2 t_{j}+s_{j}\right.$ ) and $r=s_{j} / 2$, for some large $j$, then the H-chain for $B(z, r)$ must pass through $T_{j}$, and so it includes balls of radius comparable with $r^{N}$.

Gehring and Martio [GM] show that Euclidean Hölder domains are bounded; see also the results of Gotoh [G1, Section 6]. We now give an H-chain version of this.

Proposition 3.2. Any H-chain set with parameters $J, K, L, B\left(x_{*}, r_{*}\right)$ has diameter at most $(8 K-1) r_{*}$.

Proof. Let $\Omega$ be an H-chain set with the indicated parameters, and let us fix a ball $B(x, r) \subset \Omega$, and let $\left(B_{i}\right)_{i=0}^{k}$ be a minimal length H-chain for $B(x, r)$. By $(3.1)$, we see that

$$
d\left(x, x_{*}\right)+r_{*} \leq 2 \sum_{i=0}^{k} r_{i} \leq 4 r_{*} \sum_{i=0}^{k} 2^{-(k-i) / K} \leq \frac{4 r_{*}}{1-2^{-1 / K}} \leq 8 K r_{*} .
$$

We next intend to show that if $(X, d)$ is a metric space, and $\Omega \subsetneq X$ is an H-chain set, then $\partial \Omega$ is mean porous, as defined by Koskela and Rohde $[\mathrm{KR}]$. Let us begin by defining mean porosity 
for a bounded measurable subset $E$ of $X$; it is convenient to normalize $d$ so that $\operatorname{diam}(E) \leq 1$. Suppose that $0<\delta<1<D$ and $n_{0} \in \mathbb{N}$. For every $x \in E$ and $n \in \mathbb{N}$, we write

$$
\begin{aligned}
A_{j}(x) & =\left\{y \in X: D^{-j}<d(x, y) \leq D^{-j+1}\right\}, \\
\chi_{j}(x) & = \begin{cases}1, & \text { if } \exists y \in(X \backslash E) \cap A_{j}(x): d(y, E)>\delta(D-1) d(x, y) \\
0, & \text { otherwise, }\end{cases} \\
\sigma_{n}(x) & =\sum_{j=1}^{n} \chi_{j}(x) .
\end{aligned}
$$

Then $E$ is $\left(D, \delta, n_{0}\right)$-mean porous if $\sigma_{n}(x)>n / 2$ for all $n \geq n_{0}$ and all $x \in E$.

The parameter $D$ in the above definition corresponds to $1+\epsilon$ in $[\mathrm{KR}]$, where it is assumed that $\epsilon \leq 1$. By contrast, we shall typically choose $D$ to be rather large.

Theorem 3.3. Suppose that $(X, d)$ is a metric space, and that $\Omega$ is a proper open subset of $X$. If $\Omega$ is an $H$-chain set with parameters $J, K, L$, and $B_{*} \equiv B\left(z_{*}, r_{*}\right)$, for some $2 B_{*} \subset \Omega$ and $L=1$, then $\partial \Omega$ is $\left(J+2, \delta, n_{0}\right)$-mean porous, where $\delta, n_{0}$ depend only on $J, K$, and $r_{*} / \operatorname{diam}(\Omega)$. Furthermore, if $\mu$ is a doubling measure on $X$ with doubling constant $C_{\mu}$, then $\Omega$ is an OLD set with parameters $\delta, K$ dependent only on $J, K, r_{*} / \operatorname{diam}(\Omega)$, and $C_{\mu}$.

The last statement in Theorem 3.3 is roughly analogous to the fact that in the EuclideanLebesgue setting, the boundary of a Hölder domain in $\mathbb{R}^{n}$ has Minkowski dimension less than $n$. This was proved by Jones and Makarov [JM] in the planar simply-connected space, by Smith and Stegenga [SS2] in $\mathbb{R}^{n}$, and was sharpened (for $n \geq 3$ ) and generalized to mean porous sets by Koskela and Rohde [KR]; see also [B2] where doubling measures in Euclidean space are considered. A closer analogy to these earlier results would be to say that if $E=\partial \Omega$ is the boundary of a Hölder domain or of a bounded mean porous set, and if $E_{r}$ denotes the $r \operatorname{diam}(E)$-neighborhood of $E$, then $\mu\left(E_{r}\right) \leq K r^{-\delta} \mu\left(E_{1}\right), 0<r<1$; this is true and can be proved in the same manner. However, we shall need to know that the measure of the part of $\Omega$ close to $X \backslash \Omega$ is small, not just the part close to $\partial \Omega$.

For the proof of Theorem 3.3, we need two preliminary results. The first is a variant of a rather well-known type of lemma. We omit the proof, which amounts to the boundedness of the maximal operator on $L^{p}(X), 1<p \leq \infty$, with the norm estimates that one gets from the standard proof of this fact using the Marcinkiewicz Interpolation Theorem; for other variants of this lemma, see $[\mathrm{Bo}]$ and $[\mathrm{B} 2]$.

Lemma 3.4. Suppose that $1 \leq k, R<\infty$ and that $\mathcal{F}$ is a family of balls in a doubling space $X$. Then there exists a constant $C$ dependent only on $C_{\mu}$ and $R$ such that

$$
\left\|\sum_{B \in \mathcal{F}} \chi_{R B}\right\|_{L^{k}(X)} \leq C k\left\|\sum_{B \in \mathcal{F}} \chi_{B}\right\|_{L^{k}(X)}
$$

The second result needed in the proof of Theorem 3.3 is the following lemma which generalizes to the context of H-chain sets a theorem of Smith and Stegenga [SS1, Theorem 3] (see also [G2]) for the quasihyperbolic geodesic in a Euclidean Hölder domain; this relationship is somewhat clouded by the difference between the language of minimal length $\mathrm{H}$-chains and that of quasihyperbolic geodesics. 
Lemma 3.5. Suppose that $(X, d)$ is a metric space, and that $\Omega$ is a proper open subset of $X$. If $\Omega$ is an $H$-chain set with parameters $J, K, L$, and $B_{*} \equiv B\left(z_{*}, r_{*}\right)$ and $\left\{B_{j}=B\left(z_{j}, r_{j}\right)\right\}_{j=0}^{k}$ is a minimal length $H$-chain for some ball in $\Omega$, then for each $0 \leq j \leq k, k-j \leq K \log _{2}\left(4 K r_{*} / \sum_{i=0}^{j} r_{i}\right)$.

Proof. Using (3.1), we see that

$$
\sum_{i=0}^{j} r_{i} \leq 2 r_{*} \sum_{i=0}^{j} 2^{-(k-i) / K} \leq 2 r_{*} \frac{2^{-(k-j) / K}}{1-2^{-1 / K}} \leq 4 K r_{*} 2^{-(k-j) / K}
$$

This is equivalent to the desired inequality.

Proof of Theorem 3.3. We normalize $d$ so that $\operatorname{diam}(\Omega)=1$. We may assume that $2 B_{*} \subset \Omega$, $r_{*} \leq 1 / 2$ (otherwise replace $B_{*}$ by $(1 / 2) B_{*}$ ). We first tackle the mean porosity of $\Omega$. For this part of the proof, we write $D=J+2$ and we may assume that $\partial \Omega \neq \emptyset$. Let us fix $x \in \partial \Omega$ and $n \geq n_{0} \equiv 4\left\lceil 1+\log _{D}\left(1 / r_{*}\right)\right\rceil$, and let $A_{j}(x), 1 \leq j \leq n$, be the annuli in the definition of mean porosity.

We first show that there exists a point $y \in \Omega$ such that $d(x, y) \in\left(D^{-n-1}, D^{-n}\right]$ that possesses a "short" H-chain. We choose any $x^{\prime} \in \Omega$ with $d\left(x, x^{\prime}\right) \leq D^{-n-2}$. Let $\left\{B_{i}^{\prime}=B\left(z_{i}^{\prime}, r_{i}^{\prime}\right): 0 \leq i \leq k^{\prime}\right\}$ be a minimal length $\mathrm{H}$-chain for $B\left(x^{\prime}, d_{\Omega}\left(x^{\prime}\right) / L\right)$. Since $x \notin \Omega \supset B_{i}^{\prime}$, it follows that $r_{i}^{\prime} \leq d\left(z_{i}^{\prime}, x\right)$ and, since balls $B_{i}$ and $B_{i+1}$ overlap,

$$
d\left(z_{i+1}^{\prime}, x\right) \leq d\left(z_{i+1}^{\prime}, z_{i}^{\prime}\right)+d\left(z_{i}^{\prime}, x\right)<r_{i+1}^{\prime}+r_{i}^{\prime}+d\left(z_{i}^{\prime}, x\right) \leq \operatorname{Dd}\left(z_{i}^{\prime}, x\right) .
$$

Thus we can choose $y$ to be the first point $z_{j}^{\prime}$ for which $d\left(z_{j}^{\prime}, x\right)>D^{-n-1}$. Writing $r=r_{j}^{\prime}$, it follows from Lemma 3.5 that $B(y, r)$ has an H-chain of length at most $K \log _{2}\left(4 K D^{n+2} r_{*}\right)$.

Let $\left\{B_{i}=B\left(z_{i}, r_{i}\right): 0 \leq i \leq k\right\}$ be a minimal length $\mathrm{H}$-chain for $B(y, r)$. We shall show that most of the annuli $A_{j}(x)$ contain a positive but suitably bounded number of the $z_{i}$ 's. Positivity for $j \in \mathbb{N} \cap\left[n_{0} / 4, n\right]$ follows rather easily. Since $j \leq n$, we have $d(y, x) \leq D^{-j}$. On the other hand, since $j \geq n_{0} / 4>1+\log _{D}\left(1 / 2 r_{*}\right)$, it follows that

$$
D^{-j+1}<2 r_{*} \leq d_{\Omega}\left(x_{*}\right) \leq d\left(x_{*}, x\right)
$$

Thus the H-chain $\left\{B_{i}\right\}$ must "pass through" the annular region $A_{j}(x)$. But, as with the chain for $x^{\prime}$, we have $r_{i} \leq d\left(z_{i}, x\right)$ and $d\left(z_{i+1}, x\right) \leq \operatorname{Dd}\left(z_{i}, x\right)$, and so for each $j \in\left[n_{0} / 4, n\right]$, there exists $i \in(0, k)$ such that $z_{i} \in A_{j}(x)$.

Now $k \leq K \log _{2}\left(4 K D^{n+2} r_{*}\right)<K\left(n+\log _{2} K+4\right) \log _{2} D$ and, for an appropriate constant $c^{\prime}$, $n+\log _{2} K+4 \leq c^{\prime}\left\lfloor n-n_{0} / 4\right\rfloor$. A simple counting argument thus implies that numerically less than one in three of the annuli $A_{j}(x), n_{0} / 4 \leq j \leq n$, contain $N \equiv 3 K c^{\prime} \log _{2} D$ or more points $z_{i}$; we call annuli containing at least $N$ centers bad annuli and all others good annuli. For each good annulus $A_{j}(x)$, define an index $i_{j} \in(0, k)$ so that $z_{i_{j}} \in A_{j}(x)$ and $r_{i} \leq r_{i_{j}}$ whenever $z_{i} \in A_{j}(x)$. Since $n-n_{0} / 4 \geq 3 n / 4$, more than half of all annuli $A_{j}(x), 1 \leq j \leq n$, are good annuli and $r_{i_{j}} \geq(D-1) D^{-j} / 2 N$. Since $B_{i_{j}} \subset \Omega$, we see that $\partial \Omega$ satisfies a $\left(D, 1 / 2 N D, n_{0}\right)$-mean porosity condition. 
It remains to prove the decay estimate for the measure of $\Omega_{r}=d_{\Omega}^{-1}((0, r))$. We choose $D=2 J+4$. Suppose that $n \geq n_{0}$ and $x^{\prime} \in \Omega_{D^{-n-2}} \backslash \Omega_{D^{-n-3}}$, where $n_{0} \equiv 4\left\lceil 1+\log _{D}\left(1 / r_{*}\right)\right\rceil$. As before we write

$$
A_{j}\left(x^{\prime}\right)=\left\{y \in X: D^{-j}<d\left(x^{\prime}, y\right) \leq D^{-j+1}\right\} .
$$

We pick $x \in X \backslash \Omega$ such that $d\left(x, x^{\prime}\right) \leq D^{-n-2}$. Let $\left\{B_{i}^{\prime}=B\left(z_{i}^{\prime}, r_{i}^{\prime}\right): 0 \leq i \leq k^{\prime}\right\}$ be a minimal length H-chain for $B\left(x^{\prime}, d_{\Omega}\left(x^{\prime}\right) / L\right)$. Then $r_{i}^{\prime} \leq d\left(z_{i}^{\prime}, x\right) \leq d\left(z_{i}^{\prime}, x^{\prime}\right)+D^{-n-2}$ and so

$$
d\left(z_{i+1}^{\prime}, x^{\prime}\right) \leq d\left(z_{i+1}^{\prime}, z_{i}^{\prime}\right)+d\left(z_{i}^{\prime}, x^{\prime}\right)<r_{i+1}^{\prime}+r_{i}^{\prime}+d\left(z_{i}^{\prime}, x^{\prime}\right) \leq(J+2)\left(d\left(z_{i}^{\prime}, x^{\prime}\right)+D^{-n-2}\right) .
$$

Choosing $y$ to be the first point $z_{j}^{\prime}$ for which $d\left(z_{j}^{\prime}, x^{\prime}\right)>D^{-n-1}$, and $r=r_{j}^{\prime}$, it follows that $d\left(z_{j-1}^{\prime}, x^{\prime}\right)>D^{-n-2}$ and hence that $d\left(y, x^{\prime}\right) \leq D^{-n}$. By Lemma 3.5, $y$ has an H-chain of length at most $K \log _{2}\left(4 K D^{n+1} r_{*}\right)$.

Let $\left\{B_{i}=B\left(z_{i}, r_{i}\right): 0 \leq i \leq k\right\}$ be a minimal length H-chain for $B(y, r)$. Now $n_{0} / 4 \geq$ $1+\log _{D}\left(1 / r_{*}\right)$, and so if $j \geq n_{0} / 4$, then

$$
d\left(x_{*}, x^{\prime}\right) \geq d_{\Omega}\left(x_{*}\right)-d_{\Omega}\left(x^{\prime}\right) \geq 2 r_{*}-D^{-n-2}>r_{*} \geq D^{-j+1} .
$$

Thus the H-chain must "pass through" $A_{j}\left(x^{\prime}\right)$ for every $j \in\left[n_{0} / 4, n\right]$. As before, we see that $d\left(z_{i+1}, x^{\prime}\right)<(J+2)\left(d\left(z_{i}, x^{\prime}\right)+D^{-n-2}\right)$, and so $d\left(z_{i+1}, x^{\prime}\right) \leq D^{-n-1}$ if $d\left(z_{i}, x^{\prime}\right) \leq D^{-n-2}$, while $d\left(z_{i+1}, x^{\prime}\right)<D d\left(z_{i}, x^{\prime}\right)$ otherwise. Arguing as in the mean porosity part of the proof, we see that there are at least $n / 2 \operatorname{good}$ indices $j \in[n / 4, n]$ for which there exist points $w_{j} \equiv z_{i_{j}} \in A_{j}\left(x^{\prime}\right)$ with

$$
d_{\Omega}\left(w_{j}\right)>(D-1) d\left(w_{j}, x^{\prime}\right) / 2 N D
$$

for some appropriate positive number $1<N<\infty$.

We write $\mathcal{B}=\left\{B\left(v, d_{\Omega}(v) / 20\right): v \in \Omega_{1}\right\}$. Using Lemma 1.1, we extract a countable pairwise disjoint subfamily $\mathcal{F}$ of $\mathcal{B}$ such that $\bigcup_{B \in \mathcal{F}} 5 B=\Omega$. Our first aim is to show that the sum $S \equiv \sum_{B \in \mathcal{F}} \chi_{C_{1} B}$ of characteristic functions is bounded below by $c_{1}\left(1+\log r^{-1}\right)$ on $\Omega_{r}, 0<r<1$; here, $C_{1}=50 N D /(D-1)+5$ and $c_{1}>0$ are constants dependent untimately only on $J$ and $K$. More precisely, we claim that $S\left(x^{\prime}\right) \geq n / 4$, for our arbitrary point $x^{\prime} \in \Omega_{D^{-n-2}} \backslash \Omega_{D^{-n-3}}$ and $n \geq n_{0}$.

Let $j \in[n / 4, n]$ be a good index and let $B^{j} \equiv B\left(v_{j}, d_{\Omega}\left(v_{j}\right) / 20\right) \in F$ be such that $w_{j} \in 5 B^{j}$. Since $d\left(v_{j}, w_{j}\right)<d_{\Omega}\left(v_{j}\right) / 4$, we have

$$
d_{\Omega}\left(w_{j}\right)+d_{\Omega}\left(v_{j}\right) / 4>d_{\Omega}\left(v_{j}\right)>d_{\Omega}\left(w_{j}\right)-d_{\Omega}\left(v_{j}\right) / 4,
$$

and so

$$
4 d_{\Omega}\left(w_{j}\right) / 3>d_{\Omega}\left(v_{j}\right)>4 d_{\Omega}\left(w_{j}\right) / 5 .
$$

By (3.6) and half of (3.7), we see that $x^{\prime} \in C_{1} B^{j}$.

It would follow that $S\left(x^{\prime}\right) \geq n / 2$, which is stronger than our claim, if we could show that balls $B^{j}$ corresponding to distinct good indices are distinct. However this might not be the case for successive good indices $j, j+1$ since $w_{j}$ and $w_{j+1}$ might be rather close to each other. We therefore thin out the collection of indices a little by choosing a maximal subcollection $j_{1}, j_{2}, \ldots, j_{l}$ of the good indices that satisfy $j_{i+1} \geq j_{i}+2$ for each value of $i<l$; we call the indices in this 
subcollection better indices. Clearly there are at least $n / 4$ better indices and so the claim follows if we show that the balls $B^{j}$ corresponding to distinct better indices are distinct. Suppose therefore that $j^{\prime}<j$ are any pair of better indices, and so $j \geq j^{\prime}+2$. Now,

$$
d\left(w_{j}, x\right) \leq d\left(w_{j}, x^{\prime}\right)+d\left(x^{\prime}, x\right)<D^{-j+1}+D^{-n-2}<2 D^{-j+1} .
$$

By (3.7),

$$
d\left(w_{j^{\prime}}, v_{j^{\prime}}\right)<d_{\Omega}\left(v_{j^{\prime}}\right) / 4<d_{\Omega}\left(w_{j^{\prime}}\right) / 3<\left(d\left(w_{j^{\prime}}, x^{\prime}\right)+D^{-n-2}\right) / 3
$$

and so

$$
\begin{aligned}
d\left(v_{j^{\prime}}, w_{j}\right) & >d\left(w_{j^{\prime}}, x^{\prime}\right)-d\left(w_{j^{\prime}}, v_{j^{\prime}}\right)-d\left(w_{j}, x^{\prime}\right) \\
& >2 d\left(w_{j^{\prime}}, x^{\prime}\right) / 3-D^{-n-2} / 3-2 D^{-j+1}>D^{-j^{\prime}} / 3 .
\end{aligned}
$$

Combining (3.8) and (3.9), we see that

$$
d_{\Omega}\left(v_{j^{\prime}}\right)<d\left(v_{j^{\prime}}, w_{j}\right)+d\left(w_{j}, x\right)<2 d\left(v_{j^{\prime}}, w_{j}\right)
$$

from which it follows that $w_{j} \notin B^{j^{\prime}}$. Thus $B^{j^{\prime}}$ and $B^{j}$ are distinct, as required.

We finish the proof by using a similar argument to that in [KR]. Since $S(y) \gtrsim \log r^{-1}$ for $y \in \Omega_{r}$, it suffices to find $a>0$, dependent only on allowed parameters, such that

$$
\int_{\Omega_{1}} \exp (a S(y)) d \mu(y) \lesssim \mu\left(\Omega_{1}\right), \quad 0<r<1
$$

Clearly,

$$
\int_{\Omega_{1}} e^{a S} d \mu \leq \sum_{k \geq 0} \int_{\Omega_{1}} \frac{(a S)^{k}}{k !} d \mu \leq \mu\left(\Omega_{1}\right)+\sum_{k>0} \frac{a^{k}}{k !} \int_{\Omega_{1}}\left(\sum_{B \in \mathcal{F}} \chi_{C_{1} B}\right)^{k} d \mu .
$$

By Lemma 3.4, there exists a constant $C$ dependent only on $C_{1}$ and $C_{\mu}$ such that

$$
\begin{aligned}
\int_{\Omega_{1}} e^{a S(y)} d \mu(y) & \leq \mu\left(\Omega_{1}\right)+\sum_{k>0} \frac{(a C k)^{k}}{k !} \int_{\Omega_{1}}\left(\sum_{B \in \mathcal{F}} \chi_{B}\right)^{k} d \mu \\
& \lesssim \mu\left(\Omega_{1}\right)\left(1+\sum_{k>0} \frac{(a C k)^{k}}{k !}\right) .
\end{aligned}
$$

This last series converges for all $a<1 / C e$, and so we are done.

In the above proof, we employed a "big $D$ " method to prove mean porosity and outer layer decay. Since it may be useful elsewhere, let us sketch a "small $D$ " method which is more consistent with the method of $[\mathrm{KR}]$ but does not work in the full generality considered above. For this alternative method to work we need to assume that any ball containing points on both sides of an open annular region must also contain a point inside the annulus; this is of course true if balls are assumed to be connected. We also need to assume that $\Omega$ is an H-chain set with parameter 
$L>1$, rather than merely $L \geq 1$ as above; this is not a serious restriction since, as mentioned previously, we can always take $L=2$ if $\Omega$ is a Hölder domain.

For this alternative method, we take $D=1+\epsilon$, where $\epsilon=1 / C K$ and $C$ is a sufficiently large, but universal, constant. Given $x \in \partial \Omega$, and a large $n \in \mathbb{N}$, we find as before a point $y \in \Omega$ with a short H-chain such that $d(x, y)$ is less than, but comparable to, $D^{-n}$. For appropriate $C$, the number of balls in a suitable $\mathrm{H}$-chain for $B\left(y, d_{\Omega}(y) / 2\right)$ is less than $n / 4$, say. One can then deduce that for at least half of the integers $1 \leq j \leq n$, there exists a chain ball $B_{i_{j}}$ that has points on both sides of $A_{j}(x)$ and so cannot be very small. By our extra assumption, it contains a point in $A_{j}(x)$ whose distance to the boundary is comparable to the radius of the ball (since $L>1$ ). Mean porosity now follows more or less as before.

Let us finish this section by briefly discussing the relationship between, on the one hand, the OLD and H-chain conditions and, on the other, the ADP and chain conditions introduced in [B1]. The ADP condition, defined only for balls, says that the $\mu$-measure of an annular region $B(x, R) \backslash B(x, R-r)$ is at most $(K r / R)^{\delta} \mu(B(x, R))$ for some $K, \delta$. Since $d_{B(x, R)}(y) \geq R-d(x, y)$, ADP is a stronger condition than OLD. The two conditions are equivalent if this inequality is an equality, as is the case for balls in Euclidean and many length spaces. However, it is not difficult to give examples of non-ADP balls that are OLD.

The notion in [B1] of a chain ball in a metric space $(X, d)$, is easily seen to be equivalent to the following definition of a J-chain ball (modulo a quantitatively controlled change of parameters); our new terminology is inspired by the fact that this condition is similar to the classical notion of a John domain (although neither condition implies the other). A ball $B(Z, R) \subset X$ is a $J$-chain ball, with parameters $\alpha, \beta>1$, if for every $x \in B(Z, R)$ there is an integer $k=k(x) \geq 0$ and a chain of balls $B_{i}=B\left(z_{i}, r_{i}\right), 0 \leq i \leq k$, with the following properties:

(i) $B_{0}=B(x,(R-d(x, Z)) / \beta)$ and $B_{k}=B(Z, R / 2)$;

(ii) $B_{i} \cap B_{i+1}$ is non-empty, $0 \leq i<k$;

(iii) $x \in \alpha B_{i}, 0 \leq i \leq k$;

(iv) $r_{i}=\left(R-d\left(z_{i}, Z\right)\right) / \beta, 0 \leq i<k$.

A J-chain ball contains chains satisfying the following assumptions (possibly with different $\alpha, \beta)$, where $c>0, \rho>1$ are fixed constants depending on $\alpha, \beta$ :

(ii') $B_{i} \cap B_{i+1}$ contain a ball of radius $\operatorname{cr}\left(B_{i}\right), 0 \leq i<k$;

(v) $r_{i} \geq c \rho^{i}\left(R-d\left(x, z_{0}\right)\right), 0 \leq i \leq k$.

To get $\left(\right.$ ii $\left.^{\prime}\right)$, we simply replace $\beta$ by $(1+\beta) / 2$ above, and alter the chain radii accordingly. To make (v) true, we need to make two types of changes. First, we must "thin out" the chain so that $d\left(z_{i}, z_{j}\right) \gtrsim r_{i}, 0<i<j<k$; this can be done without affecting the other conditions, except for a controlled change in $c$. The doubling condition then implies that after a bounded number of steps we reach a ball $B_{i_{1}}$, with $r_{i_{1}} / r_{0}>2$. This process cannot immediately be iterated, since the points $z_{i}$ may wander back near $x$ for large $i$, thus leading to small values of $r_{i}$. We therefore discard the balls $B_{i}, i>i_{1}$ and append the full J-chain for $z_{i_{1}}$ before iterating this whole process; we leave the details to the reader (see also [BKL2]). Thus we may define a J-chain ball as a ball satisfying (i), (ii'), (iv), and (v) for appropriate $c>0$ and $\alpha, \beta, \rho>1$; (iii) follows from the other conditions. With this new definition, it is easy to see that J-chain balls are H-chain sets (with $L=\beta$ ). In the converse direction, it is not hard to come up with examples of balls which are $\mathrm{H}$-chain sets but not J-chain balls (this is analogous to the fact that Hölder domains are a strictly larger class than John domains). 


\section{Local John-Nirenberg: Sufficiency}

Smith and Stegenga [SS2] and Hurri-Syrjänen $[\mathrm{H}]$ proved that in the Euclidean-Lebesgue setting, a necessary and sufficient condition for the inequality $\|\cdot\|_{E I(\Omega)} \leq C\|\cdot\|_{B M O(\Omega)}$ to hold is that $\Omega$ is a Hölder domain. In this section, we shall strengthen the sufficiency part of this result; necessity is left for the next section. Throughout this section, $(X, d, \mu)$ is a doubling space with doubling constant $C_{\mu}$.

Theorem 4.1. If $\Omega \subsetneq X$ is an $H$-chain set, with $H$-chain parameter $L=2$, then $B M O_{\text {loc }}(\Omega) \subset$ $E I(\Omega)$. Furthermore, $\|\cdot\|_{E I(\Omega)} \leq C\|\cdot\|_{B M O_{\mathrm{loc}}(\Omega)}$, where $C$ depends only on $C_{\mu}$ and the H-chain parameters $J, K$, and $r_{*} / \operatorname{diam}(\Omega)$.

Given an open set $\Omega \subset X$ with a distinguished ball $B_{*}$, let $G B M O(\Omega)\left(\operatorname{resp} . G B M O_{\text {loc }}(\Omega)\right)$ be the space of all $u \in B M O(\Omega)\left(\right.$ resp. $\left.B M O_{\text {loc }}(\Omega)\right)$ such that

$$
\exists C \in \mathbb{R}: \quad 2 B \subset \Omega, r(B) \leq \operatorname{diam}(\Omega) \Longrightarrow\left|u_{B}-u_{B_{*}}\right| \leq C\left(1+\log _{2} \frac{\operatorname{diam}(\Omega)}{r(B)}\right) .
$$

Let $\|u\|_{G B M O(\Omega)}\left(\operatorname{resp} .\|u\|_{G B M O_{\mathrm{loc}}(\Omega)}\right)$ be either $\|u\|_{B M O(\Omega)}\left(\right.$ resp. $\left.\|u\|_{B M O_{\mathrm{loc}}(\Omega)}\right)$ or the smallest $C$ for which the above condition is valid, whichever is larger. Note that the choice of $B_{*}$ can affect the norms $\|u\|_{G B M O(\Omega)}$ and $\|u\|_{G B M O_{\text {loc }}(\Omega)}$, but leaves the sets $G B M O(\Omega)$ and $G B M O_{\text {loc }}(\Omega)$ unchanged. If $\Omega$ is an $\mathrm{H}$-chain set, we implicitly choose the $G B M O$ ball $B_{*}$ to equal the $\mathrm{H}$-chain ball $B_{*}$.

It is easily verified that if $u \in E I(\Omega) \cap B M O(\Omega)$ then $u \in G B M O(\Omega)$ (with control of norms once $B_{*}$ is fixed). Thus we can split the local John-Nirenberg imbedding is equivalent to the combination of the following pair of imbeddings:

$$
\begin{aligned}
\|\cdot\|_{G B M O(\Omega)} & \leq C_{1}\|\cdot\|_{B M O(\Omega)}, \\
\|\cdot\|_{E I(\Omega)} & \leq C_{2}\|\cdot\|_{G B M O(\Omega)} .
\end{aligned}
$$

Let us also label the (more) local versions of these inequalities:

$$
\begin{aligned}
\|\cdot\|_{G B M O_{\mathrm{loc}}(\Omega)} & \leq C_{1}\|\cdot\|_{B M O_{\mathrm{loc}}(\Omega)}, \\
\|\cdot\|_{E I(\Omega)} & \leq C_{2}\|\cdot\|_{G B M O_{\mathrm{loc}}(\Omega)}
\end{aligned}
$$

The H-chain condition is tailor-made to give (4.2) and $\left(4.2^{\prime}\right)$, as we shall now see.

Proposition 4.4. If $\Omega \subsetneq X$ is an $H$-chain set, then (4.2) holds; if the $H$-chain parameter $L$ is at least 2 , then $\left(4.2^{\prime}\right)$ also holds. The parameter $C_{1}$ depends only on $C_{\mu}, r_{*} / \operatorname{diam}(\Omega)$, and the $H$-chain parameters $J, K$.

Proof. We normalise $d$ so that $\operatorname{diam}(\Omega)=1$. Because neighbouring H-chain balls overlap on a ball of comparable radius, we can prove as in Lemma 2.1 that there exists a constant $C$ dependent only on $C_{\mu}$ and the H-chain parameter $J$ such that $\left|u_{B}-u_{B_{*}}\right| \leq C k\|u\|_{B M O(\Omega)}$, where $k$ is the length of the H-chain from a ball $B$ satisfying $2 B \subset \Omega$ to the distinguished ball $B_{*}$. H-chain Property (b) now implies the required result. Inequality $\left(4.2^{\prime}\right)$ is handled similarly.

In the case of a length space, we can strengthen Proposition 4.4 as follows. 
Theorem 4.5. Suppose that $d$ is a length metric on $X$ and that $\Omega \subsetneq X$ is open. Then an inequality of type (4.2) or (4.2') is true if and only if $\Omega$ is an H-chain set (and we may take $L=2$ ). If $\Omega \subset X$ is a ball then (4.2) is true with $C_{1}=C_{\mu}$.

To prove this theorem, we need to show that the H-chain condition is necessary, a task we postpone until the next section, and we also need the sharpened estimate in the last statement. For the latter, we fix an arbitrary ball $B=B(Z, R)$. The required estimate follows from Lemma 2.1 if we construct a nesting chain $B\left(z_{i}, r_{i}\right), i=0, \ldots, k \equiv\left\lfloor\log _{2}(R / r)\right\rfloor+1$ in $B(Z, R)$ from $B_{0}=B(x, r)$ to $B_{k}=B(Z, R)$, whenever $B(x, 2 r) \subset B(Z, R)$. The length condition ensures the existence, for every $\epsilon>0$ and $y, y^{\prime} \in X$, of paths $\gamma_{y, y^{\prime}}^{(\epsilon)}$ connecting $y$ and $y^{\prime}$, with arclength no more than $d\left(y, y^{\prime}\right)+\epsilon$; we assume without loss of generality that these paths are parametrized by arclength. Let $z_{0}=x$, let $r_{i}=2^{i} r$ for all $0 \leq i<k$, and let $r_{k}=R$. It remains to define the points $z_{i}, 0<i \leq k$. Given $z_{i-1}$, let $z_{i}=Z$ if $Z \in B_{i-1}$; otherwise, we choose $z_{i}=\gamma_{z_{i-1}, Z}^{(r / k)}\left(r_{i}\right)$. These balls may "creep" closer to $X \backslash B(X, R)$, but the choice of $\epsilon$ ensures that they all remain within $B(Z, R)$. We leave to the reader the routine verification that this chain has the required properties.

Theorem 4.6. If $\Omega \subsetneq X$ is a $(\delta, K)$-OLD set, then (4.3') holds for some $C_{2}$ dependent only on $C_{\mu}, \delta$, and $K$.

Theorem 4.6, together with Proposition 4.4 and Theorem 3.3, implies Theorem 4.1. In the case of a length space, it can be strengthened as follows.

Theorem 4.7. Suppose that $d$ is a length metric on $X$ and that $\Omega \subsetneq X$ is open. Then an inequality of type (4.3) or (4.3') is true if and only if $\Omega$ is an $O L D$ set. In particular, if $B \subset X$ is a ball then (4.3') is true with $C_{2}$ dependent only on $C_{\mu}$.

Since length space are BOLD sets with universal constants, the only part of Theorem 4.7 that does not follow readily from Theorem 4.6 is the necessity of the OLD condition. We postpone the proof of this until the next section.

As discussed in Section 3, length spaces are BH-chain sets, and in them the concepts of Hchain sets and Hölder domains coincide. Thus Theorems 4.5 and 4.7 imply Theorem 0.4 - in fact we may replace $B M O(\Omega)$ with $B M O_{\mathrm{loc}}(\Omega)$ (and similarly for the norms). Theorem 0.3 follows easily from this stronger version of Theorem 0.4 , since if $u \in B M O_{\mathrm{loc}}(\Omega)$, then $u \in B M O_{\mathrm{loc}}(B)$ for any $B \subset \Omega$, and the resulting exponential integrability over $B$ implies the boundedness of $f_{B}\left|u-u_{\Omega}\right| d \mu$.

For the proof of Theorem 4.6, we need the following Calderón-Zygmund decomposition. This lemma and Lemma 2.3 are quite different in one important respect: all balls $B_{j, k}$ below are elements of a single decomposition, whereas the balls $B_{j}^{m}$ in Lemma 2.3 form a sequence of nested decompositions indexed by $m$.

Lemma 4.8. Suppose that $\Omega$ is a proper open subset of $X$ with diameter $0<R<\infty$ and distinguished sub-ball $B_{*}$. Suppose further that $u \in G B M O_{\mathrm{loc}}(\Omega)$ with $u_{B_{*}}=0$, and that $\beta \geq$ $\|u\|_{G B M O_{\mathrm{loc}}(\Omega)}$. For $k \in \mathbb{N}$, let $A_{k}=d_{\Omega}^{-1}\left(\left(2^{-k} R, 2^{-k+1} R\right]\right)$ and $\widetilde{A}_{k}=d_{\Omega}^{-1}\left(\left(2^{-k-1} R, 2^{-k+2} R\right)\right)$. 
Then there exists a collection of pairwise disjoint balls $\left\{B_{j, k}=B\left(z_{j, k}, r_{j, k}\right)\right\}$ where $(j, k)$ ranges over some subset of $\mathbb{N} \times \mathbb{N}$ such that for all such pairs $(j, k)$,

(i) $8 B_{j, k} \subset \widetilde{A}_{k}$ and $r_{j, k} \leq d_{\Omega}\left(z_{j, k}\right) / 32$;

(ii) $(k+5) \beta<\left|u_{B_{j, k}}\right| \leq(k+6) \beta$;

(iii) $\left|u_{8 B_{j, k}}\right| \leq(k+5) \beta$;

(iv) $|u(x)| \leq(k+5) \beta$, for almost all $x \in A_{k} \backslash \bigcup_{i} 5 B_{i, k}$.

Proof. The annuli $A_{k}$ partition $\Omega$, so we can define the indicator function $n(x)=k$ for $x \in A_{k}$. For any ball $B=B(x, r), x \in \Omega$, define $a(B)=\left|u_{B}\right| /(n(x)+5) \beta$. Let us normalise $d$ so that $R=1$. We shall inductively define families $\mathcal{G}_{i} \supset \mathcal{G}_{i-1}, i \in \mathbb{N}$, of balls $B(x, r), x \in \Omega$, $r \leq d_{\Omega}(x) / 32$, from which we define a residual set $S_{i}=\Omega \backslash \bigcup_{B \in \mathcal{G}_{i}} 5 B$. We begin by setting $\mathcal{G}_{0}=\emptyset$. Inductively for all $i \in \mathbb{N}$, let

$$
\begin{aligned}
\mathcal{B}_{i} & =\left\{B\left(x, 2^{-i-4} d_{\Omega}(x)\right): x \in S_{i-1}\right\} \\
\widetilde{\mathcal{B}}_{i}^{\prime} & =\left\{B \in \mathcal{B}_{i}: a(B)>1\right\}
\end{aligned}
$$

and let $\mathcal{F}_{i}$ be a countable pairwise disjoint subset of $\widetilde{\mathcal{B}}_{i}$ such that the 8-dilates of the balls in $\mathcal{F}_{i}$ cover the union of the balls in $\widetilde{\mathcal{B}}_{i}$. We complete the inductive step by defining $\mathcal{G}_{i}$ to be $\mathcal{G}_{i-1} \cup \mathcal{F}_{i}$. To finish our construction, we define $\mathcal{G}=\bigcup_{i=1}^{\infty} \mathcal{G}_{i}, S=\bigcap_{i=1}^{\infty} S_{i}$, and list all elements $B(x, r)$ of $\mathcal{G}$ in the form $\left\{B_{j, k}\right\}$, where $k=n(x)$, and $j$ is simply a counter.

Since $\log _{2}\left(1 / d_{\Omega}(x)\right)<n(x)$ and $u \in G B M O(\Omega)$, we have $a(B) \leq 1$ for all balls $B \equiv B(x, r)$, $d_{\Omega}(x) / 16 \leq r \leq d_{\Omega}(x) / 2$. With this hint, the proof that the balls $B_{j, k}$ have the desired properties is very similar to that in Lemma 2.3 , so we leave the details to the reader.

Proof of Theorem 4.6. Normalize $u$ so that $u_{B_{*}}=0$. Let $\alpha \equiv 6 C_{\mu}\|u\|_{B M O_{\mathrm{loc}}(\Omega)}$ and $\beta \equiv$ $\|u\|_{G B M O_{\text {loc }}(\Omega)}$. The conclusion is readily deduced from the estimate

$$
\begin{aligned}
\mu\left(S_{m}\right) & \equiv \mu(\{x \in \Omega:|u(x)|>m(\alpha+2 \beta)\}) \\
& \leq\left[2^{(6-2 m) \delta} K+2^{-1} 3^{-m} C_{\mu}^{3-m}\right] \mu(\Omega) .
\end{aligned}
$$

Let $\left\{B_{j, k}\right\}$ and $A_{k}$ be as in Lemma 4.8 for $\beta \equiv\|u\|_{G B M O_{\mathrm{loc}}(\Omega)}$. Since $16 B_{j, k} \subset \Omega$, we can apply (2.5) with $\alpha \equiv 6 C_{\mu}\|u\|_{B M O_{\mathrm{loc}}(\Omega)}$ to get that

$$
\mu\left(\left\{x \in 8 B_{j, k}:\left|u(x)-u_{B_{j, k}}\right|>m \alpha\right\}\right) \leq \frac{\mu\left(8 B_{j, k}\right)}{2\left(3 C_{\mu}\right)^{m}} \leq \frac{\mu\left(B_{j, k}\right)}{2 \cdot 3^{m} C_{\mu}^{m-3}} .
$$

Lemma 4.8(ii) now implies that

$$
\mu\left(\left\{x \in 8 B_{j, k}:|u(x)|>m(\alpha+2 \beta)\right\}\right) \leq \mu\left(B_{j, k}\right) / 2 \cdot 3^{m} C_{\mu}^{m-3}, \quad k \leq 2 m-6,
$$

and so, writing $\Omega^{m}=\bigcup_{k=2 m-5}^{\infty} A_{k}$, we have

$$
\mu\left(\left\{x \in \Omega \backslash \Omega^{m}:|u(x)| \leq m(\alpha+2 \beta)\right\}\right) \leq \mu(\Omega) / 2 \cdot 3^{m} C_{\mu}^{m-3} .
$$


But $\Omega$ is $(\delta, K)$-OLD, and so $\mu\left(\Omega^{m}\right) \leq 2^{(6-2 m) \delta} K$. Combining this with (4.10), we deduce $(4.9)$.

\section{Local John-Nirenberg: Necessity}

In Section 4, we saw that a local John-Nirenberg inequality holds for all H-chain sets. In this section we examine the necessity of the H-chain condition (and of OLD for (4.3)). We begin with a pair of examples, which show that the local John-Nirenberg inequality is true for certain sets that are not H-chain (or even OLD) sets, but false for other such sets (even for balls). We use notation such as $B M O_{X}(\Omega)$ to indicate $B M O(\Omega)$ with respect to the ambient space $X$.

Example 5.1. First, we define a discrete metric subspace $Y$ of $\left(\mathbb{R}^{2}, d\right)$ where $d$ is the "square metric" given by $d(a, b)=\max \left(\left|a_{1}-b_{1}\right|,\left|a_{2}-b_{2}\right|\right)$, and subscripts denote coordinates. The set $Y$ has as elements the origin and points $x_{i}=\left(1-2^{-i-1}, 2^{-i}\right), y_{i}=\left(1-d_{i}, 2^{-i}\right)$, and $z_{i}=\left(1+d_{i}, 2^{-i}\right)$, $i \in \mathbb{N}$, where $0<d_{i} \leq 4^{-i-1}$. We define a Borel measure $\mu$ on $Y$ by the equations $\mu(\{a\})=w(a)$, $a \in X$, where $w: Y \rightarrow \mathbb{R}$ is defined by the equations $w(0)=1, w\left(x_{i}\right)=w\left(y_{i}\right)=w\left(z_{i}\right)=2^{-i}$. It is clear that $Y$ is a doubling space, as is its subspace $X$ which contains the origin and the points $x_{i}, z_{i}$ only.

Let $B_{X} \equiv B_{X}(0,1)$ and $B_{Y} \equiv B_{Y}(0,1) . B_{X}$ is an H-chain set in $X$, and so $B M O_{X}\left(B_{X}\right) \subset$ $E I\left(B_{X}\right)$. On the other hand, $B_{Y}$ is not an H-chain set in $Y$, since any chain of intersecting balls in $B_{Y}$ from $B_{Y}\left(y_{i}, d_{i}\right)$ to $B_{Y}(0,1 / 2)$ must involve a transition from a ball around $y_{i}$ of radius at most $2 d_{i} \leq 4^{-i}$ to an intersecting ball around another point of radius exceeding $2^{-i-1}-d_{i}$. Additionally, if $d_{i}=4^{-i^{2}-1}$, then $B_{Y}$ is not even an OLD set.

However, regardless of the choise of $d_{i} \leq 4^{-i-1}$, we claim that $B M O_{Y}\left(B_{Y}\right) \subset E I\left(B_{Y}\right)$. Suppose that $u \in B M O_{Y}\left(B_{Y}\right)$, where we may normalise $\|u\|_{B M O_{Y}\left(B_{Y}\right)}=1$. If $x \in X$ and $r>0$, then the only difference between balls $B_{Y}(x, r)$ and $B_{X}(x, r)$ is that $B_{Y}(x, r)$ might contain some points $y_{i} \notin B_{X}(x, r)$; in any such case, the partner point $x_{i}$ lies in both balls. Thus the measures of the two balls differ by a factor at most 2. Additionally, $B_{Y}\left(x_{i}, 2^{-i-1}\right)=\left\{x_{i}, y_{i}\right\}$, and so $\left|u\left(x_{i}\right)-u\left(y_{i}\right)\right| \leq 2$. It follows that $\left|u_{B_{Y}(x, r)}-u_{B_{X}(x, r)}\right| \leq 2$ and that $\|u\|_{B M O_{X}\left(B_{X}\right)} \leq$ $2(1+2)=6$. Similarly, the consequent exponential integrability of $\left|u-u_{B_{X}}\right|$ on $B_{X}$ easily implies the exponential integrability of $\left|u-u_{B_{Y}}\right|$ on $B_{Y}$, thus proving our claim.

We note one interesting feature of the space $X$. As already noted $B M O\left(B_{X}\right) \subset E I\left(B_{X}\right)$. However $B M O_{\mathrm{loc}}=L^{1}(X)$, a much bigger space than $B M O\left(B_{X}\right)$, since if $B$ is a ball containing $x_{i}, i \in \mathbb{N}$, and $2 B \subset B_{X}$, then $B=\left\{x_{i}\right\}$.

Example 5.2. Let $Z=Y \backslash\left\{x_{i}: i \in \mathbb{N}\right\}$, where $Y$ is as in Example 5.1 with $d_{i}=4^{-i-1}$. The ball $B_{Z} \equiv B_{Z}(0,1)$ is an OLD, but not an H-chain, set. Defining $u(0)=0$ and $u\left(y_{i}\right)=2^{i} / i^{2}, i \in \mathbb{N}$, it is easy to see that $u \in B M O_{Z}\left(B_{Z}\right) \backslash G B M O_{Z}\left(B_{Z}\right)$.

The pathology in Example 5.1 is due to the fact that $B_{Y}$ is a rather unpleasant ball. Our first theorem in this section says that if balls are uniformly H-chain sets then the H-chain condition is a necessary condition for the first part of the John-Nirenberg inequality. Since balls in a length space are uniformly $\mathrm{H}$-chain sets with $L=2$, this proves necessity in Theorem 4.5. For the rest of this section, $(X, d, \mu)$ is a doubling space with doubling constant $C_{\mu}$. 
Theorem 5.3. Suppose that $\Omega \subsetneq X$ is a BH-chain set with parameters $J, K$, L, and that (4.2) holds for some constant $C_{1}$. Then $\Omega$ is an H-chain set with parameters $J, K^{\prime}, L$, and $B_{*} \equiv$ $B\left(z_{*}, r_{*}\right)$, with $K^{\prime}$ dependent only on $C_{1}, C_{\mu}, J, K$, and $r_{*} / \operatorname{diam}(\Omega)$. A similar result is true for (4.2') as long as $L \geq 2$.

Proof. We look only at $(4.2)$ as $\left(4.2^{\prime}\right)$ is similar. Let $L^{\prime}=\max \{L, 2\}$, choose $z_{*} \in \Omega$, and let $r_{*}=$ $d_{\Omega}\left(z_{*}\right) / L^{\prime}$. For any open $U \subset \Omega$, define a function $k_{U}: U \times U \rightarrow \mathbb{Z}_{+}$, by letting $k_{U}(x, y)$ be the length of the shortest $\mathrm{H}$-chain (with respect to $U$ ) with parameters $J, L$, and $B_{*}=B\left(y, d_{U}(y) / L^{\prime}\right)$, for the ball $B\left(x, d_{U}(x) / L^{\prime}\right)$, if such an H-chain exists; otherwise, let $k_{U}(x, y)=\infty$. We now define $u(x) \equiv u_{N}(x)=\min \left\{k_{\Omega}\left(x, x_{*}\right), N\right\}$, where $N$ is an arbitrary but fixed positive number.

It is a routine matter to check that if $\Omega$ does not satisfy the conclusion of the theorem, then $\left\|u_{N}\right\|_{G B M O(\Omega)} \rightarrow \infty(N \rightarrow \infty)$. Thus our theorem reduces to the claim that $\|u\|_{B M O(\Omega)}$ depends only on allowed parameters; most importantly, it is independent of $N$. If $x \in B(Z, R) \subset \Omega$, then the H-chain condition for $B(Z, R)$ implies that

$$
\begin{aligned}
|u(Z)-u(x)| & \leq\left|k_{\Omega}\left(Z, x_{*}\right)-k_{\Omega}\left(x, x_{*}\right)\right| \\
& \leq k_{B(Z, R)}(x, Z) \leq K \log \left(4 R / d_{B(Z, R)}(x)\right) .
\end{aligned}
$$

Let $\mathcal{B}=\left\{B\left(x, d_{B(Z, R)}(x) / 10\right): x \in B(Z, R)\right\}$, and extract a countable pairwise disjoint subcollection $\mathcal{F} \subset \mathcal{B}$ whose 5 -dilates cover $B(Z, R)$. For all $m \in \mathbb{N}$, we let $A_{m}=d_{B(Z, R)}^{-1}\left(\left(2^{-m} R, 2^{1-m} R\right]\right)$, $\widetilde{A}_{m}=d_{B(Z, R)}^{-1}\left(\left(2^{-1-m} R, 2^{2-m} R\right)\right)$, and $\mathcal{F}_{m}$ be the family of balls in $\mathcal{F}$ whose centers lie in $A_{m}$. The families $\mathcal{F}_{m}$ partition $\mathcal{F}$, and $\bigcup_{B \in \mathcal{F}_{m}} B \subset \widetilde{A}_{m}$. Now, (5.4) implies that $\left|u_{5 B}-u_{B(Z, R / 2)}\right| \lesssim m$ for all $B \in \mathcal{F}_{m}$, and so

$$
\int_{B(Z, R)}\left|u-u_{B(Z, R / 2)}\right| d \mu \lesssim \sum_{m=1}^{\infty} m \mu\left(\widetilde{A}_{m}\right)
$$

Since $B(Z, R)$ is $\left(\delta, K_{1}\right)$-OLD, where $\delta, K_{1}$ depend only on $J, K$, and $C_{\mu}$, we have $\mu\left(\widetilde{A}_{m}\right) \leq$ $\left(K_{1} 2^{2-m}\right)^{\delta}$. Thus $f_{B(Z, R)}\left|u-u_{B(Z, R / 2)}\right| d \mu \lesssim 1$.

We now prove an analogous result for the necessity of the OLD condition in the containment $G B M O(\Omega) \subset E I(\Omega)$; this proves necessity in Theorem 4.7.

Theorem 5.5. Suppose that $\Omega \subsetneq X$ is a bounded $(\delta, K)$-BOLD set, and that (4.3) holds for some constant $C_{2}$. Then $\Omega$ is a $\left(\delta^{\prime}, K^{\prime}\right)$-OLD set, with $\delta^{\prime}, K^{\prime}$ dependent only on $C_{2}, C_{\mu}, \delta$, and $K$.

Proof. Without loss of generality, we assume that $\operatorname{diam}(\Omega)=1$. For all $k \in \mathbb{N}$, let

$$
u_{k}(x)=\max \left\{\log \left[d_{\Omega}(x)\right]^{-1}, k\right\}, \quad x \in \Omega, k \in \mathbb{N},
$$

and $A_{k}=d_{\Omega}^{-1}\left(\left(2^{-k-1}, 2^{-k}\right]\right)$. Since balls are uniformly OLD sets and

$$
\left|u_{k}(x)-u_{k}(z)\right| \leq \log \left(r / d_{B(z, r)}(x)\right), \quad x \in B(z, r) \subset \Omega,
$$


it follows that $f_{B(z, r)}\left|u_{k}-\left(u_{k}\right)_{B(z, r / 2)}\right| d \mu$ is uniformly bounded for all $B(z, r) \subset \Omega$ and all $k \in \mathbb{N}$. Thus $\left\|u_{k}\right\|_{B M O(\Omega)}$ is uniformly bounded, and it is also easy to deduce that $\left\|u_{k}\right\|_{G B M O(\Omega)}$ is uniformly bounded for any fixed choice of distinguished sub-ball $B_{*}$ satisfying $2 B_{*} \subset \Omega$.

Suppose for the purposes of contradiction that $\Omega$ does not satisfy the conclusion of the theorem, but that there exists some fixed $c>0$ and $\left(c_{k}\right) \in \mathbb{R}$ such that the integrals $\int_{\Omega} \exp \left(c \mid u_{k}-\right.$ $\left.c_{k} \mid\right) d \mu, k \in \mathbb{N}$, have a uniform bound. The constants $c_{k}$ must be uniformly bounded-otherwise these integrals would not be uniformly bounded on any fixed ball $B$ for which $2 B \subset \Omega$. It follows that $\int_{\Omega} \exp \left(c u_{k}\right) d \mu$ is uniformly bounded. Since $\Omega$ does not satisfy the conclusion of the theorem, we can extract a sequence of annuli $A_{n_{k}}$ such that $\mu\left(A_{n_{k}}\right)>2^{-n_{k} / k}$ and so

$$
\int_{\Omega} \exp \left(c u_{n_{k}}\right) d \mu \geq \int_{A_{n_{k}}} \exp \left(c u_{n_{k}}\right) d \mu>2^{-n_{k} / k} \cdot e^{c n_{k}} \rightarrow \infty \quad(k \rightarrow \infty) .
$$

We have yet to give an example of a ball $B$ such that $E I(B) \not \supset G B M O(B)$. Our final theorem does this, and shows that the OLD hypothesis in Theorem 4.6 is rather sharp even for balls.

Proposition 5.6. Suppose that $\left(n_{k}\right)$ is an increasing sequence of integers such that $n_{1} \geq 2$ and $n_{k+1} / n_{k} \geq[(k+1) / k]^{2}$ for all $k \in \mathbb{N}$. Then there exists a doubling space $(X, d, \mu)$ and a ball $B_{0} \subsetneq X$ with the following properties:

(a) $\mu\left(A_{n_{k}}\right)=k 2^{-n_{k} / k} \mu\left(B_{0}\right)$, where $A_{k} \equiv d_{B_{0}}^{-1}\left(\left(2^{-k-1}, 2^{-k}\right]\right), k \in \mathbb{N}$.

(b) $G B M O\left(B_{0}\right) \backslash E I\left(B_{0}\right)$ is non-empty.

Proposition 5.6 shows that the implication " $G B M O\left(B_{0}\right)$ implies $E I\left(B_{0}\right)$ " is false for certain balls which fail the OLD hypothesis at arbitrarily slow rates. In fact if $B_{0}$ is a non-OLD set, then by definition there exists an increasing sequence of integers $\left(n_{k}\right)$ such that $\mu\left(A_{n_{k}}\right) \geq$ $k 2^{-n_{k} / k} \mu\left(B_{0}\right)$. Without loss of generality, we may assume that $n_{1} \geq 2$ and $n_{k+1} / n_{k} \geq[(k+1) / k]^{2}$ (these conditions are in any case added only for technical reasons). Imposing equality in the measure estimate merely slows down the failure rate.

Proof of Proposition 5.6. The required metric space $(X, d)$ will be a discrete subspace of $\left(\mathbb{R}^{2}, d\right)$, where $d(a, b)=\left|a_{1}-b_{1}\right|+\left|a_{2}-b_{2}\right|$, and $X$ consists of points $x_{0}=(0,0), x_{k}=\left(1-2^{-n_{k}-2}, 2^{-n_{k}}\right)$, $k \in \mathbb{N}$, together with certain other points $y_{k, j}, k \in \mathbb{N}, j=0, \ldots, j_{k}$, to be defined later. Clearly every $x_{k}$ lies in $B_{0}=B\left(x_{0}, 1\right)$; the points $y_{k, j}$ will be chosen to lie in $2 B_{0} \backslash B_{0}$.

Define $\mu$ by the equations $\mu(\{z\})=w(z), z \in X$, where $w: X \rightarrow[0,1]$. Normalizing $\mu\left(B_{0}\right)=1$, the hypotheses imply that $w\left(x_{k}\right)=k 2^{-n_{k} / k}, k \in \mathbb{N}$, and $w\left(x_{0}\right)=1-\sum_{k=1}^{\infty} w\left(x_{k}\right)$. The assumptions on $\left(n_{k}\right)$ ensure that $n_{k} \geq 2 k^{2}$ and so $w(0)$ is bounded away from zero-in fact, $w(0) \geq 1-\sum_{k=1}^{\infty} k 2^{-2 k}=5 / 9$.

Note that $\left(w\left(x_{k}\right)\right)$ is a decreasing sequence. For each $k \in \mathbb{N}$, let $j_{k}=\left\lfloor\log _{2}\left(w\left(x_{k-1}\right) / w\left(x_{k}\right)\right)\right\rfloor \geq$ $0, y_{k, j}=\left(1+2^{j-n_{k}-2}, 2^{-n_{k}}\right)$, and $w\left(y_{k, j}\right)=2^{j} w\left(x_{k}\right)$. The points $y_{k, j}$ fulfil two roles. Their primary purpose is to provide stepping-stones so that, as $r$ increases, the measure of $B\left(x_{k}, r\right)$ increases by bounded factors; without them, $\mu$ would not be doubling. If we order the points $y_{k, j}$ by their indices in increasing lexicographical order, then the jump in measure from any such point to the next one is a factor between 1 and 2, and the points are also spaced supergeometrically since the distance from $y_{k-1,0}$ to $y_{k, j_{k}}$ is at least $2^{-n_{k-1}-1}$ for all $k>1$. With these observations, it is easy to show that $X$ is a doubling space. 
A secondary purpose of the points $y_{k, j}$ is to ensure that if $B\left(x_{k}, r\right) \subset B(0,1), k \in \mathbb{N}$, then $B\left(x_{k}, r\right)=\left\{x_{k}\right\}$; this follows because $d\left(x_{k}, y_{k}\right)=2^{-n_{k}-1}<\min \left\{d\left(x_{k}, x_{j}\right): j \in \mathbb{N}, j \neq k\right\}$. Consequently, $B M O\left(B_{0}\right)=L^{1}\left(B_{0}\right)$. Defining $u: B(0,1) \rightarrow \mathbb{R}$ by $u\left(x_{0}\right)=0$ and $u\left(x_{k}\right)=n_{k}$, $k \in \mathbb{N}$, it follows that $u \in G B M O\left(B_{0}\right)$. However, $u \notin E I\left(B_{0}\right)$, since $\mu(\{x \in B(0,1):|u(x)|>$ $\left.n_{k}\right\} \geq k 2^{-n_{k} / k}, k \in \mathbb{N}$.

Let us consider two special cases of the above proposition which relate to comments in the introduction. Taking $n_{k}=2 k^{3}$, the ball $B_{0}$ (with the restricted $d$ and $\mu$ attached) is not a doubling space, since $\mu\left(B_{B_{0}}\left(x_{k}, 2 r\right)\right)$ is much larger than $\mu\left(B_{B_{0}}\left(x_{k}, r\right)\right)$ if $k$ is large and $r=2^{-2(k-1)^{3}-1}$. If we take instead $n_{k}=2 k^{2}$, then $G B M O_{X}\left(B_{0}\right) \backslash E I\left(B_{0}\right)$ is non-empty, even though $B_{0}$ is now a doubling subspace of $X$; the function $u$ constructed in the proof lies in $G B M O_{X}\left(B_{0}\right)$ but cannot lie in $G B M O_{B_{0}}\left(B_{0}\right) \subset E I\left(B_{0}\right)$. In fact, it is easy to see directly that $u \notin B M O_{B_{0}}\left(B_{0}\right)$ by considering $B\left(x_{k}, r_{k}\right)=\left\{x_{k}, x_{k+1}\right\}$, where $r_{k}=d\left(x_{k}, x_{k+2}\right)$.

\section{REFERENCES}

[Bo] B. Bojarski, Remarks on Sobolev imbedding inequalities, Proc. of the conference on Complex Analysis, Joensuu 1987, Lecture Notes in Math. 1351, Springer-Verlag, Berlin, 1989, pp. 52-68.

[B1] S.M. Buckley, Is the maximal function of a Lipschitz function continuous?, Ann. Acad. Sci. Fenn., to appear.

[B2] S.M. Buckley, Strong doubling conditions, Math. Ineq. Appl. 1 (1998), 533-542.

[BKL1] S.M. Buckley, P. Koskela, and G. Lu, Subelliptic Poincaré inequalities: the case $p<1$, Publ. Mat. 39 (1995), 313-334.

[BKL2] S.M. Buckley, P. Koskela, and G. Lu, Boman equals John, Proc. XVIth Rolf Nevanlinna Colloquium, de Gruyter, Berlin, 1996, pp. 91-99.

[CW1] R. Coifman and G. Weiss, Analyse harmonique non-commutative sur certains espaces homogènes, Lecture Notes in Math. 242, Springer-Verlag, Berlin, 1971.

[CW2] R. Coifman and G. Weiss, Extensions of Hardy spaces and their use in analysis, Bull. Amer. Math. Soc. 83 (1977), 569-645.

[GN] N. Garofalo and D.-M. Nhieu, Isoperimetric and Sobolev inequalities for Carnot-Carathéodory spaces and existence of minimal surfaces, Comm. Pure Appl. Math. 49 (1996), 1081-1144.

[GM] F.W. Gehring and O. Martio, Lipschitz classes and quasiconformal mappings, Ann. Acad. Sci. Fenn. Ser. A. I. Math. 10 (1985), 203-219.

[G1] Y. Gotoh, On global integrability of BMO functions on general domains, J. Anal. Math. 75 (1998), 67-84.

[G2] Y. Gotoh, On domains with some growth conditions for quasihyperbolic metric, preprint.

[GS] J. Graczyk and S. Smirnov, Collett, Eckmann and Hölder, Invent. Math. 133 (1998), 69-96.

[HK] P. Hajłasz and P. Koskela, Sobolev met Poincaré, to appear in Mem. Amer. Math. Soc..

[HKM] J. Heinonen, T. Kilpeläinen, and O. Martio, Nonlinear potential theory of degenerate elliptic equations, Oxford Univ. Press, Oxford, 1993.

[HS] I. Holopainen and P.M. Soardi, A strong Liouville theorem for p-harmonic functions on graphs, Ann. Acad. Sci. Fenn. Ser. A. I. Math. 22 (1997), 205-226.

[H] R. Hurri-Syrjänen, The John-Nirenberg inequality and a Sobolev inequality for general domains, J. Math. Anal. Appl. 175 (1993), 579-587.

[JN] F. John and L. Nirenberg, On functions of bounded mean oscillation, Comm. Pure Appl. Math. 14 (1961), 415-426.

[J] P.W. Jones, Extension theorems for BMO, Indiana Univ. Math. J. 29 (1980), 41-66.

[JM] P.W. Jones and N.G. Makarov, Density properties of harmonic measure, Ann. Math. 142 (1995), 427-455. 
[K] P. Koskela, Old and new on the quasihyperbolic metric, Quasiconformal mappings and analysis (Ann Arbor, MI, 1995), Springer, New York, 1998, pp. 205-219.

[KR] P. Koskela and S. Rohde, Hausdorff dimension and mean porosity, Math. Ann. 309 (1997), 593-609.

[NSW] A. Nagel, E.M. Stein, and S. Wainger, Balls and metrics defined by vector fields I: basic properties, Acta Math. 155 (1985), 103-147.

[RR] H.M. Reimann and T. Rychener, Funktionen beschränkter mittelerer Oszillation, Lecture Notes in Math. 489, Springer, Berlin, 1975.

[RL] L. Ruilin and Y. Lo, BMO functions in spaces of homogeneous type, Scientia Sinica (Series A) 27 (1984), 695-708.

[SS1] W. Smith and D.A. Stegenga, Hölder domains and Poincaré domains, Trans. Amer. Math. Soc. 319 (1990), 67-100.

[SS2] W. Smith and D.A. Stegenga, Exponential integrability of the quasihyperbolic metric in Hölder domains, Ann. Acad. Sci. Fenn. Ser. A I. Math. 16 (1991), 345-360.

[S] S. Staples, $L^{p}$-averaging domains and the Poincaré inequality, Ann. Acad. Sci. Fenn. Ser. A I. Math. 14 (1989), 103-127.

[VSC] N.Th. Varopoulos, L. Saloff-Coste, and T. Coulhon, Analysis and geometry on groups, Cambridge Tracts in Math. 100, Cambridge Univ. Press, Cambridge, 1992.

[VG] S.K. Vodop'yanov and A.V. Greshnov, On extension of functions of bounded mean oscillation from domains in a space of homogeneous type with intrinsic metric, Siberian Math. J. 36 (1995), 873-901.

Department of Mathematics, National University of Ireland, Maynooth, Co. Kildare, Ireland.

E-mail address: sbuckley@maths.may.ie 